\title{
A defective dNTP pool hinders DNA replication in cell cycle-reactivated terminally differentiated muscle cells
}

\author{
Deborah Pajalunga, ${ }^{*, 1}$ Elisa Franzolin ${ }^{2}$, Martina Stevanoni ${ }^{2}$, Sara Zribi ${ }^{1}$, Nunzia Passaro', Aymone Gurtner ${ }^{3}$, Samantha Donsante ${ }^{1}$, \\ Daniela Loffredo ${ }^{1}$, Lidia Losanno ${ }^{1}$, Vera Bianchi ${ }^{2}$, Antonella Russo ${ }^{2,4}$, Chiara Rampazzo ${ }^{\star 2}$ and Marco Crescenzi ${ }^{\star 1}$
}

Terminally differentiated cells are defined by their inability to proliferate. When forced to re-enter the cell cycle, they generally cannot undergo long-term replication. Our previous work with myotubes has shown that these cells fail to proliferate because of their intrinsic inability to complete DNA replication. Moreover, we have reported pronounced modifications of deoxynucleotide metabolism during myogenesis. Here we investigate the causes of incomplete DNA duplication in cell cycle-reactivated myotubes (rMt). We find that rMt possess extremely low levels of thymidine triphosphate (dTTP), resulting in very slow replication fork rates. Exogenous administration of thymidine or forced expression of thymidine kinase increases deoxynucleotide availability, allowing extended and faster DNA replication. Inadequate dTTP levels are caused by selective, differentiation-dependent, cell cycleresistant suppression of genes encoding critical synthetic enzymes, chief among which is thymidine kinase 1 . We conclude that lack of dTTP is at least partially responsible for the inability of myotubes to proliferate and speculate that it constitutes an emergency barrier against unwarranted DNA replication in terminally differentiated cells.

Cell Death and Differentiation (2017) 24, 774-784; doi:10.1038/cdd.2017.4; published online 10 February 2017

Terminal differentiation is commonly defined as a state in which cells have permanently lost their ability to divide, while acquiring specialized properties. However, this definition is merely operational and, despite decades of study, the mechanisms that keep terminally differentiated cells tenaciously arrested are insufficiently understood. Indeed, the long-sought goal of reactivating terminally differentiated cells to proliferation is still elusive, despite its great potential in regenerative medicine. ${ }^{1}$

Skeletal muscle myotubes constitute an excellent model system for terminal differentiation and have long been studied $^{2,3}$ in a still ongoing effort to induce their proliferation. The achievement of proliferative reactivation of myotubes has been repeatedly claimed but found to be scarcely reproducible. Even the best methods devised so $\operatorname{far}^{4}$ display very low efficiency and their molecular mechanisms await elucidation.

Reactivation of the cell cycle in myotubes can be achieved by a variety of means, including infection with oncogenic viruses, ${ }^{2,3,5}$ forced expression of cell cycle genes ${ }^{6}$ or oncogenes, ${ }^{7}$ removal of cell cycle inhibitors (CKIs), ${ }^{8}$ loss of tumor suppressors such as $\mathrm{pRb},{ }^{9}$ and induction of abortive apoptosis. ${ }^{4}$ Irrespective of the triggering stimulus, cell cycle re-entry does not lead to widespread, sustained proliferation of myotube-derived cells. Rather, it generally results in apoptosis, ${ }^{10} \mathrm{G}_{2}$-phase arrest, ${ }^{6}$ and/or mitotic catastrophe, ${ }^{11}$ depending on the specific methods adopted to initiate the cell cycle. These heterogeneous outcomes might originate from a common cause; however reactivated, myotubes cannot complete DNA synthesis and suffer DNA damage. Interestingly, other non-proliferating cells (e.g., quiescent or senescent), reactivated by the same means used to force myotubes into the cell cycle, undergo complete DNA replication and proliferate extensively. Thus, deficient DNA duplication is intrinsic to myotubes and not due to the reactivating conditions. ${ }^{11}$ We have argued that defective DNA duplication and DNA damage occur in most terminally differentiated cell types upon reactivation. ${ }^{11}$ In this view, terminal differentiation entails critical, characteristic traits that hinder DNA replication and, hence, cell proliferation.

The cellular concentrations of DNA precursors, the deoxynucleoside triphosphates (dNTPs) are set by the balance between synthesis via two anabolic pathways, degradation by catabolic enzymes, and consumption by DNA polymerases. The anabolic pathways comprise de novo synthesis by ribonucleotide reductase (RNR), converting ribonucleoside diphosphates to the corresponding deoxynucleotides, and the salvage pathways, which phosphorylate deoxynucleosides.

The dNTP pool varies in size according to the functional state of the cell. ${ }^{12-14}$ It expands through the upregulation of key synthetic enzymes shortly before $S$ phase, when the need for dNTPs becomes high. ${ }^{13-16}$ During quiescence, whether permanent or reversible, dNTPs are needed only for mitochondrial replication and DNA repair, and the dNTP pool is small. ${ }^{16}$ Myotubes conform to this rule and contain small amounts of dNTPs. ${ }^{17}$ We hypothesized that incomplete DNA replication and DNA damage in rMt might stem, at least

\footnotetext{
${ }^{1}$ Department of Cell Biology and Neurosciences, Italian National Institute of Health, Rome, Italy; ${ }^{2}$ Department of Biology, University of Padua, Padua, Italy and ${ }^{3}$ Department of Research, Advanced Diagnostics and Technological Innovation, SAFU Unit, Translational Research Area, Regina Elena National Cancer Institute, Rome, Italy *Corresponding author: D Pajalunga or M Crescenzi, Department of Cell Biology and Neurosciences, Istituto Superiore di Sanità, Viale Regina Elena, 299, Rome 00156, Italy. Tel: +39 0649903164; Fax: +39 0649902040; E-mail: deborah.pajalunga@iss.it or marco.crescenzi @ iss.it

or C Rampazzo, Department of Biology, University of Padua, Via Ugo Bassi, 58B, Padua 35131, Italy. Tel: +39 0498276456; Fax: +39 0498276280;

E-mail: rampazzo@ bio.unipd.it

${ }^{4}$ Current address: Department of Molecular Medicine, University of Padua, Padua, Italy.

Received 07.7.16; revised 02.1.17; accepted 10.1.17; Edited by B Dynlacht; published online 10.2.2017
} 
partially, from a failure to expand properly the dNTP pool upon cell cycle re-entry.

Here we show that in rMt the concentration of dNTPs increases relative to control myotubes but in a highly unbalanced manner, with deoxythymidine and deoxyguanosine triphosphates (dTTP and dGTP) remaining at the very low levels associated with non-proliferation. Restoration of dNTP levels closer to those of proliferating myoblasts extends DNA replication and reduces DNA damage in rMt. Low dTTP and dGTP levels are due to persistent downregulation of genes encoding key dTTP synthetic enzymes, prominent among which is thymidine kinase 1 (TK1). Such genes appear to be permanently downregulated during terminal differentiation of myotubes and unresponsive to cell cycle reactivation. Our results unveil one mechanism through which permanent proliferation arrest is ensured in postmitotic muscle cells.

\section{Results}

Atypical response of the myotube dNTP pools to cell cycle reactivation. To determine whether deoxynucleotide scarcity is responsible for incomplete DNA replication in rMt, dNTP levels were dynamically measured in proliferating murine myoblasts and in myotubes, either resting or reactivated by RNAi to the p21 and p27 $\mathrm{CKIs}^{8}$ (Figure 1 and Supplementary Table S1). In these experiments, reactivation efficiency was about $30-40 \%$, evaluated as the percentage of cells double-positive for 5-bromo-2'-deoxyuridine (BrdU) incorporation into DNA and muscle-specific myosin heavy chain (data not shown). rMt (Figure 1, Mt p21p27i) were analyzed at different time points encompassing $\mathrm{S}$ phase. Proliferating myoblasts (Prol Mb) displayed a typical balance among the dNTPs, with dTTP as the largest and dGTP as the smallest pool, ${ }^{13}$ while resting myotubes (Mt CTRi) showed the expected low levels of all four dNTPs. ${ }^{17}$ Surprisingly, rMt showed amounts of deoxycytidine

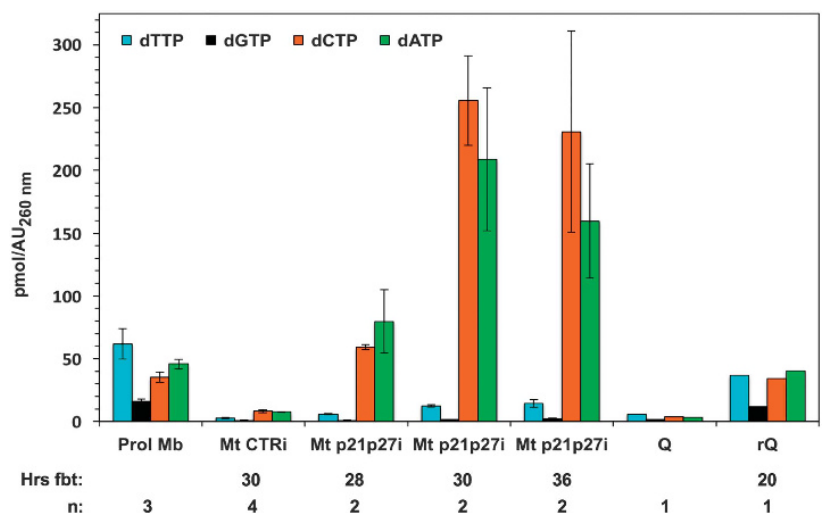

Figure 1 Reactivated myotubes possess low levels of dTTP and dGTP. Myotubes were transfected with siRNAs to p21 and p27 (Mt p21p27i) or to GFP as a control (Mt CTRi) and harvested at the indicated times from the beginning of treatment (Hrs fbt). Reference samples include proliferating myoblasts (Prol Mb), myoblasts cultured in suspension to attain quiescence $(Q)$, and formerly quiescent myoblasts reactivated by replating (rQ). The dNTP pool was extracted with $60 \%$ methanol and individual dNTPs quantitated by a DNA polymerase-based assay. The data are expressed as pmoles of deoxynucleotide per absorbance unit (AU) at $260 \mathrm{~nm}$ (see Materials and Methods section). The results are shown as averages \pm S.E.M. of the indicated numbers $(n)$ of independent experiments and deoxyadenosine triphosphate (dCTP and dATP, respectively) markedly exceeding those found in proliferating myoblasts. In striking contrast, dTTP and dGTP concentrations were almost unchanged, compared with those of resting myotubes, remaining well below those of proliferating myoblasts (Figure 1 and Supplementary Table S1). For comparison, myoblasts rendered quiescent in suspension culture $(Q)^{18}$ showed the expected low levels of all four dNTPs. ${ }^{12}$ When these cells were reactivated (rQ) by reestablishing substrate adhesion, the dNTP pools promptly returned to proliferation levels.

dNTP pool complementation extends DNA synthesis in reactivated myotubes. As $\mathrm{rMT}$ perform only incomplete DNA replication, we asked whether restoring proliferation-like levels of dTTP and dGTP would extend DNA synthesis.

In initial experiments, we attempted to increase dNTP concentrations by silencing SAMHD1, a key dNTP catabolic enzyme that coordinates the dNTP pool with the cell cycle. ${ }^{19}$ Immunoblotting demonstrated a fairly efficient knockdown, resulting in higher levels of dCTP (Supplementary Figure S1). However, the other three dNTPs were left unchanged, in contrast to what observed in other cell types. ${ }^{19,20}$ The lack of effect of SAMHD1 depletion on the dTTP and dGTP pools suggests that their shortage in myotubes is determined by deficient synthesis, rather than high catabolism.

In an alternative strategy to remodel the dNTP pool, myotubes reactivated by CKI suppression were cultured in the presence of $500 \mu \mathrm{M}$ deoxyguanosine (GdR) and/or $100 \mu \mathrm{M}$ thymidine (TdR) and compared with control rMt. These concentrations were determined empirically (see Materials and Methods section). We observed that early treatment with deoxynucleosides inhibited S-phase entry of $\mathrm{rMt}$ and found that the optimal time for addition was $17 \mathrm{~h}$ from the start of siRNA transfection, close to the beginning of $S$ phase (around $24 \mathrm{~h}$ ). Figure $2 \mathrm{a}$ and Supplementary Table S2 show that exogenous addition of $\mathrm{GdR}+\mathrm{TdR}$ or $\mathrm{TdR}$ alone increased both dGTP and dTTP, whereas the sole provision of GdR exclusively raised dGTP levels.

The extent of DNA replication was quantitated under all of the above conditions. As multinucleated myotubes are scarcely amenable to cytofluorimetric analyses, we resorted to in situ microfluorimetric measurements of DNA content in individual nuclei, as reflected by 4',6-diamidino-2-phenylindole dilactate (DAPI) fluorescence intensity. Myotubes reactivated by $\mathrm{p} 21$ and p27 RNAi (Figure $2 b$ and Supplementary Table S3, p21p27i) displayed a modest increase in DNA content (27\% above controls, Supplementary Table S3, CTRi). Strikingly, treatment with $\mathrm{GdR}+\mathrm{TdR}$ significantly extended DNA synthesis (DNA content 52\% above controls, Supplementary Table S3, p21p27i GdR+TdR).

To investigate the individual contribution of the two deoxynucleosides, we treated rMt with either GdR or TdR. Administration of $\mathrm{GdR}$, consistent with its inability to increase dTTP levels, did not meaningfully increase DNA synthesis in rMt (Figure 2c; and Supplementary Table S3, p21p27i GdR). In contrast, treatment with TdR alone, which raised both dTTP and dGTP levels, increased rMt DNA content to 56\% above control myotubes, mimicking the combined action of $\mathrm{GdR}$ + TdR (Figure 2c; Supplementary Table S3, p21p27i TdR). 
Thus administration of TdR alone is sufficient to double the extent of DNA replication in rMt.

We investigated whether improved DNA replication reduces the DNA damage that accompanies incomplete genome

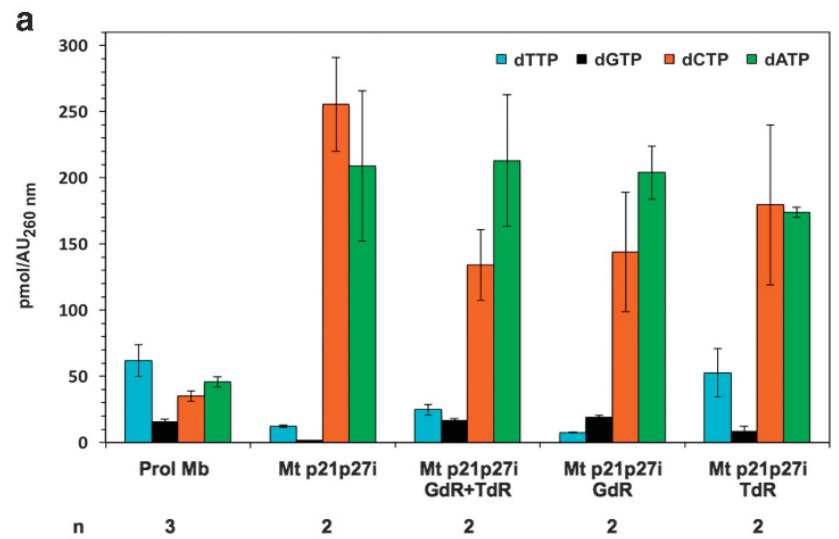

b

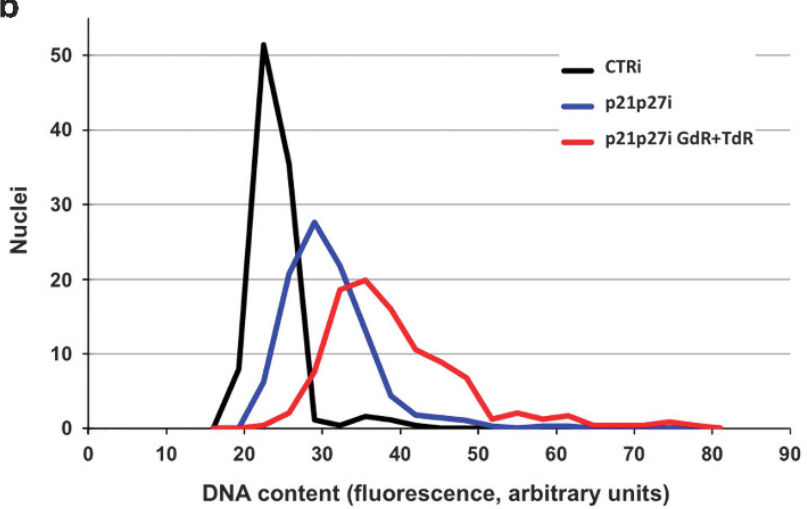

C

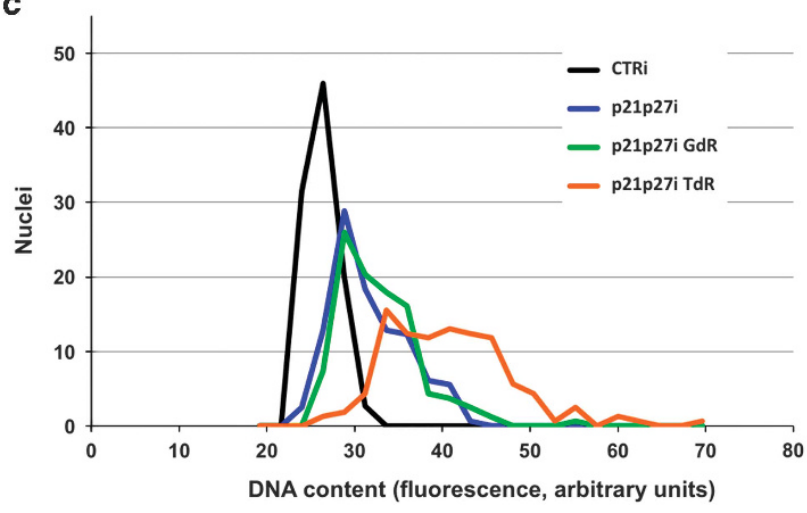

duplication in rMt. ${ }^{11}$ To this aim, we performed microfluorimetry for $\gamma-\mathrm{H} 2 \mathrm{AX}$, a marker of double-strand DNA breaks, in rMt with or without addition of GdR+TdR. Deoxynucleoside administration caused a significant reduction $(36.9 \% \pm 4.7$,

d

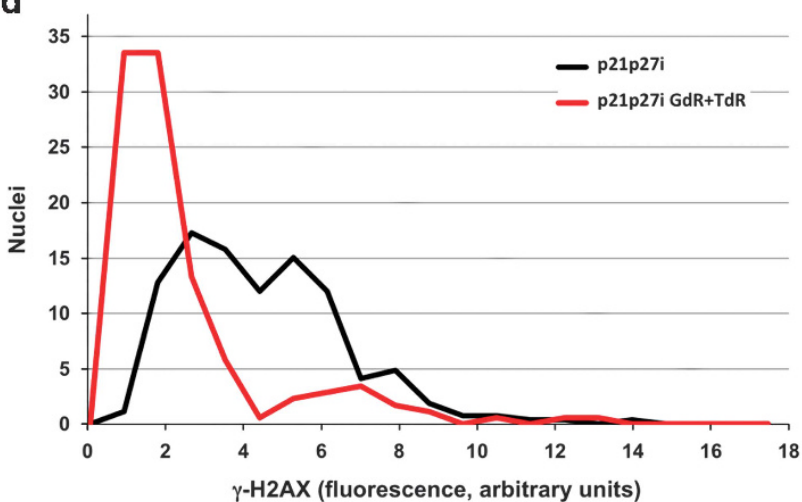

e

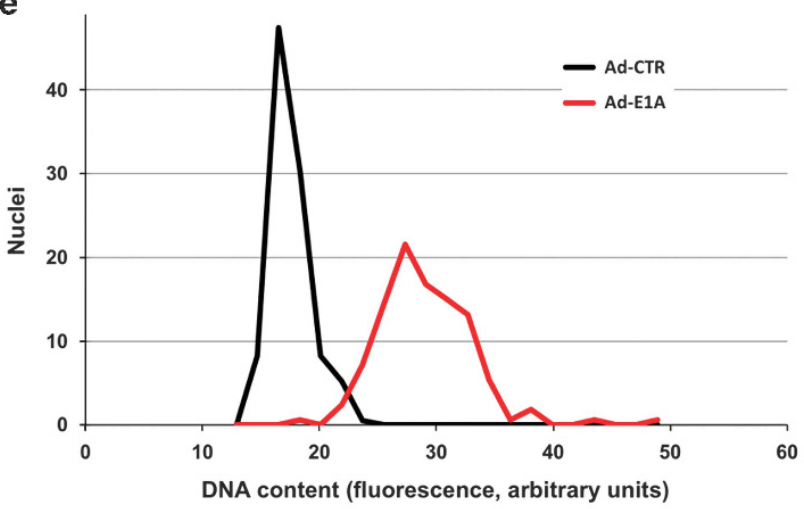

f

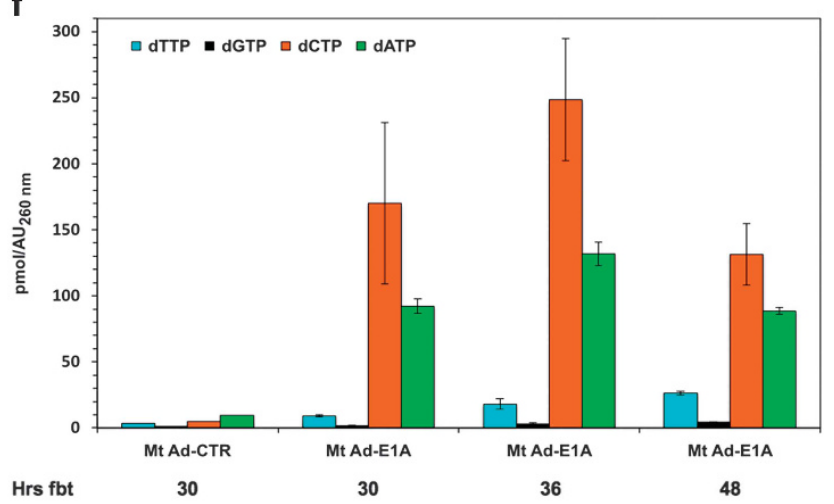

Figure 2 Higher dTTP and dGTP levels allow extended DNA replication in reactivated myotubes. (a) Myotubes reactivated by p21 and p27 RNAi as in Figure 1. GdR (500 $\mu$ M) and/or TdR $(100 \mu \mathrm{M})$ were added to the culture medium $17 \mathrm{~h}$ later. The dNTP pool was quantitated as in panel (a) $30 \mathrm{~h}$ from the beginning of treatment (fbt); data from 2-4 independent experiments and statistical evaluations are shown in Supplementary Table S2. Values for proliferating myoblasts and myotubes reactivated by p21 and p27 RNAi (30 h fbt) are the same shown in Figure 1 and are reproduced here for ease of reference. (b and c) Myotubes were transfected as in panel (a); $30 \mathrm{~h} f b t$, at the end of $S$ phase, the cells were immunostained for MCM7, a marker of cell cycle reactivation, and counterstained with DAPI. Nuclear DNA content was evaluated by microfluorimetric measurement of DAPI fluorescence in individual $\mathrm{MCM}^{+}$myotube nuclei. On average, 209 nuclei per sample were evaluated (range 112-285) and their DNA content distribution is shown by the corresponding curve. Nuclei belonging to control, resting myotubes were uniformly MCM7 ${ }^{-}$and their DNA content was determined without selection. The graphs refer to 1 representative experiment out of 2-4; Supplementary Table S3 summarizes all the results. (d) Myotubes, treated as indicated, were subjected, $30 \mathrm{~h}$ fbt, to immunofluorescence for $\gamma-\mathrm{H} 2 \mathrm{AX}$ and Ki67, a marker of cell cycle reactivation. $\gamma-\mathrm{H} 2 \mathrm{AX}$ staining intensities were measured in Ki67-positive nuclei by microfluorimetry. Control myotubes displayed no detectable $\gamma$-H2AX fluorescence and are not reported in the graph. (e) Myotubes were infected with an E1A-expressing adenovirus (Ad-E1A) or a control one (Ad-CTR). At the end of S phase, $39 \mathrm{~h}$ postinfection, microfluorimetric quantitation of DNA content was performed as in panel (b). The graph reports one out of the three experiments; Supplementary Table S3 summarizes all the results. (f) dNTP measurements in myotubes reactivated by E1A or infected with Ad-CTR. The myotubes were harvested at the indicated times 
$n=2, \quad P<0.000001$ by Kolmogorov-Smirnov) in $\gamma$-H2AX staining (Figure 2d and Supplementary Figure S2), indicating that at least part of the damage incurred by rMt is due to deoxynucleotide shortage.

One other means to induce myotubes into the cell cycle is through expression of the adenovirus E1A oncogene. ${ }^{5}$ Microfluorimetry (Figure 2e) showed that E1A expression attains duplication of a higher proportion of the genome, compared with p21 and p27 suppression (average DNA content 49 versus $27 \%$ above controls, respectively, Supplementary Table S3). dNTP pool measurements revealed that E1A generates somewhat higher levels of both dGTP and dTTP (Figure 2f) than reactivation induced by CKI depletion (Figure 2a, Mt p21p27i). Altogether, these results lead to the conclusion that the dNTP pool limits the extent of DNA replication in rMt.

Slow replication fork rate in reactivated myotubes is partially rescued by dNTP complementation. To explore the dynamics of DNA replication in rMt, we performed DNA combing analyses of myotubes reactivated by $\mathrm{CKI}$ depletion in the presence or absence of deoxynucleoside treatment, compared with proliferating myoblasts. As illustrated in Figures $3 a$ and $b$, the replication fork rate of $r M t$ was extremely low, less than one-sixth of that measured in proliferating myoblasts (median 0.35 versus $2.33 \mathrm{~kb} / \mathrm{min}$, respectively, Supplementary Table S4), mimicking the effect of replication stress conditions. ${ }^{21}$ Addition of $\mathrm{GdR}+\mathrm{TdR}$ partially rescued replication fork rate, bringing it to $0.91 \mathrm{~kb} / \mathrm{min}$, nearly half of that of myoblasts. This result suggests that poor DNA replication in myotubes is primarily a result of the lack of building blocks.

Myoblasts and rMt supplemented with deoxynucleosides had comparable values of total DNA fiber length (median 1052.0 versus $996.6 \mathrm{~kb}$, respectively, as shown by DNA counterstaining; Supplementary Table S5). In contrast, unsupplemented rMt displayed significantly reduced fiber length (median $488.1 \mathrm{~kb} ; \quad P<0.01$ Kruskal-Wallis test; Supplementary Table S5). This observation suggests an intrinsic DNA fragility, consistent with the high prevalence of DNA breaks in these cells (Figure $2 \mathrm{~d}$ and Pajalunga et $a{ }^{11}{ }^{11}$ ). The shorter fiber length prevented the measurement of some parameters in unsupplemented rMt (Supplementary Table S4).

Consistent with the reduction in fork rates, the average distance between activated replication origins was significantly reduced in rMt dosed with deoxynucleosides, compared with proliferating myoblasts (median inter-origin distance 113.4 versus $154.8 \mathrm{~kb}$; Figure $3 \mathrm{c}$ ). This result fits the notion that, during replication stress, additional, otherwise inactive origins fire to fill in replication gaps. ${ }^{22,23}$ Smaller replication clusters are observed in deoxynucleoside-treated rMt than in myoblasts (Figure 3d).

The proportion of unidirectional forks in deoxynucleosidetreated rMt was increased, in comparison with myoblasts, with slight statistical significance $(0.05<P<0.01$, chi-square test; Supplementary Table S4). However, this parameter, in both cell types, fell within the ranges determined in other mammalian cell lines. ${ }^{24}$ No differences were observed when paused/arrested forks and asynchronous patterns were compared in deoxynucleoside-dosed rMt and myoblasts (Supplementary Table S4).
Taken together, these results suggest that the main consequence of the deficiency of dNTPs in rMt is a pronounced reduction in fork rate, which is indeed partly rescued by the addition of deoxynucleosides.

Key enzymes in thymidine nucleotide synthesis are downregulated in reactivated myotubes. To understand the genesis of the relative lack of dTTP and dGTP in rMt, we explored the expression of a number of enzymes involved in their synthesis. For this purpose, RNA extracted from proliferating myoblasts, control myotubes, or rMt was analyzed by quantitative, real-time PCR (qPCR). Figure $4 a$ shows that, as expected of non-proliferating cells, postmitotic myotubes replace the ribonucleotide reductase $\mathrm{R} 2$ subunit with p53R2. ${ }^{25}$ In agreement with our previous results, ${ }^{17}$ at least three enzymes involved in thymidine nucleotide synthesis (dCMP deaminase (DCTD); thymidylate synthase (TS); TK1) were strongly downregulated in resting myotubes. Their expression barely rose upon cell cycle re-entry, suggesting an explanation for the low levels of dTTP found in CKIsuppressed rMt. The concomitant lack of dGTP in reactivated myotubes reflects the scarcity of dTTP that is the allosteric effector of GDP reduction by ribonucleotide reductase. ${ }^{26}$

Compared with $\mathrm{CKI}$-suppressed $\mathrm{rMt}$, myotubes reactivated by E1A displayed somewhat higher expression of DCTD, TS, and $\operatorname{TK} 1$ (1.9, 1.5, and 3.9-fold, respectively; Figures 4a and b), in keeping with their higher levels of dTTP (Figure 2f). As a control, quiescent myoblasts displayed a shallower downregulation of the dTTP synthesis enzymes and, upon cell cycle reactivation, promptly returned to an expression pattern typical of proliferating cells (Figure 4c).

Altogether, these results indicate that low expression of specific biosynthetic enzymes is responsible for the limiting amount of dTTP in rMt.

DNA synthesis in reactivated myotubes critically depends on thymidine kinase activity. To determine whether the inadequate synthesis of dTTP in rMt is indeed due to low anabolism, we increased thymidine kinase activity. Myotubes reactivated by p21 and p27 knockdown were infected with a recombinant adenovirus carrying herpes simplex thymidine kinase (Ad-TK) or a control virus. In keeping with the qPCR results, TK1 activity was extremely low in postmitotic myotubes (Mt CTRi and Mt Ad-Ctr in Figure 5a) and only moderately increased in control-infected, CKI-suppressed rMt (Mt p21p27i Ad-CTR). Conversely, Ad-TK-infected myotubes showed very high TK activity, independent of cell cycle reactivation (Figure 5a, Mt Ad-TK and Mt p21p27i Ad-TK).

Ad-TK muscle cells exhibited extremely high levels of dTTP, irrespective of cell cycle re-entry (Figure 5b). Interestingly, they also showed a marked increase in dGTP, attributable to the stimulatory allosteric action of dTTP on GDP reduction by RNR. ${ }^{26}$ Despite the high concentrations of dTTP, myotubes infected with Ad-TK were reactivated by CKI suppression just as efficiently as the control-infected ones (data not shown).

As expected, control-infected rMt displayed limited DNA replication (Figure 5c and Supplementary Table S6). In contrast, in rMt forcibly expressing TK, DNA synthesis was dramatically extended, though not reaching completion. 
a

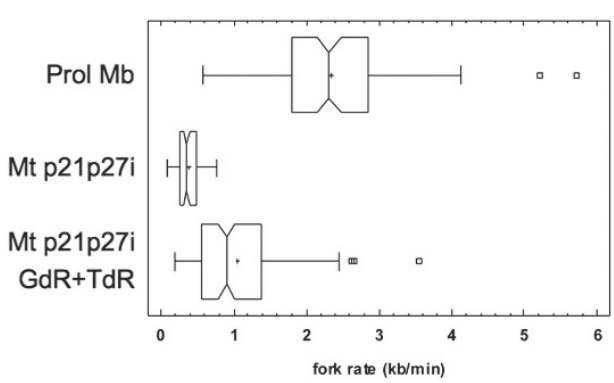

c

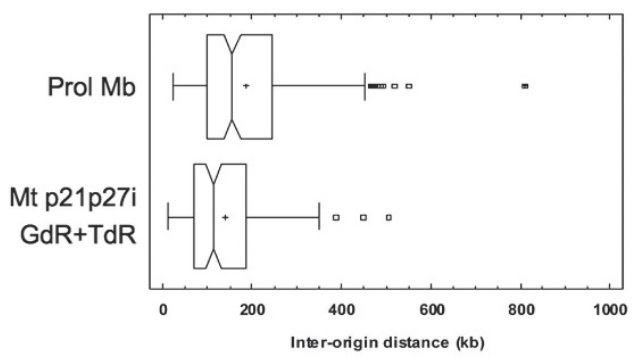

b

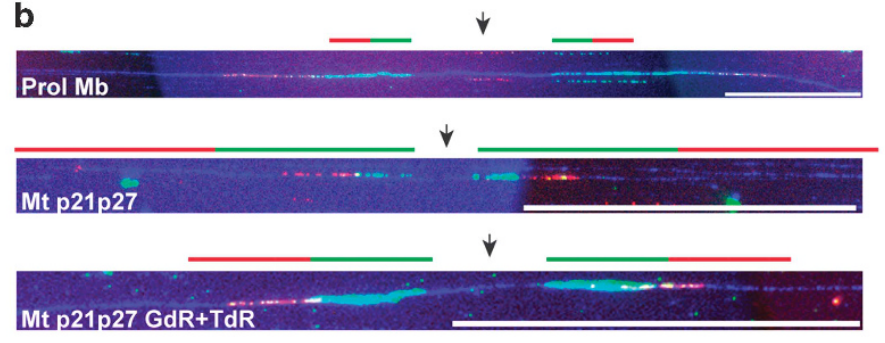

d

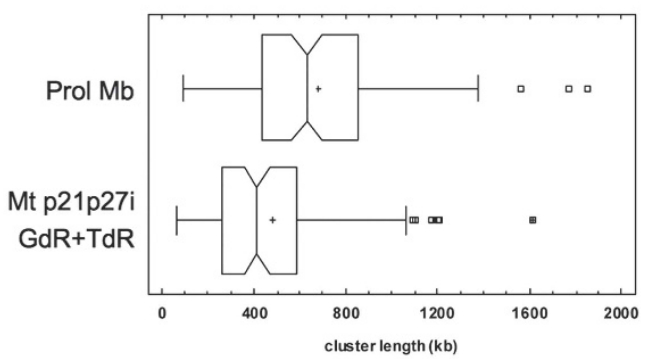

e
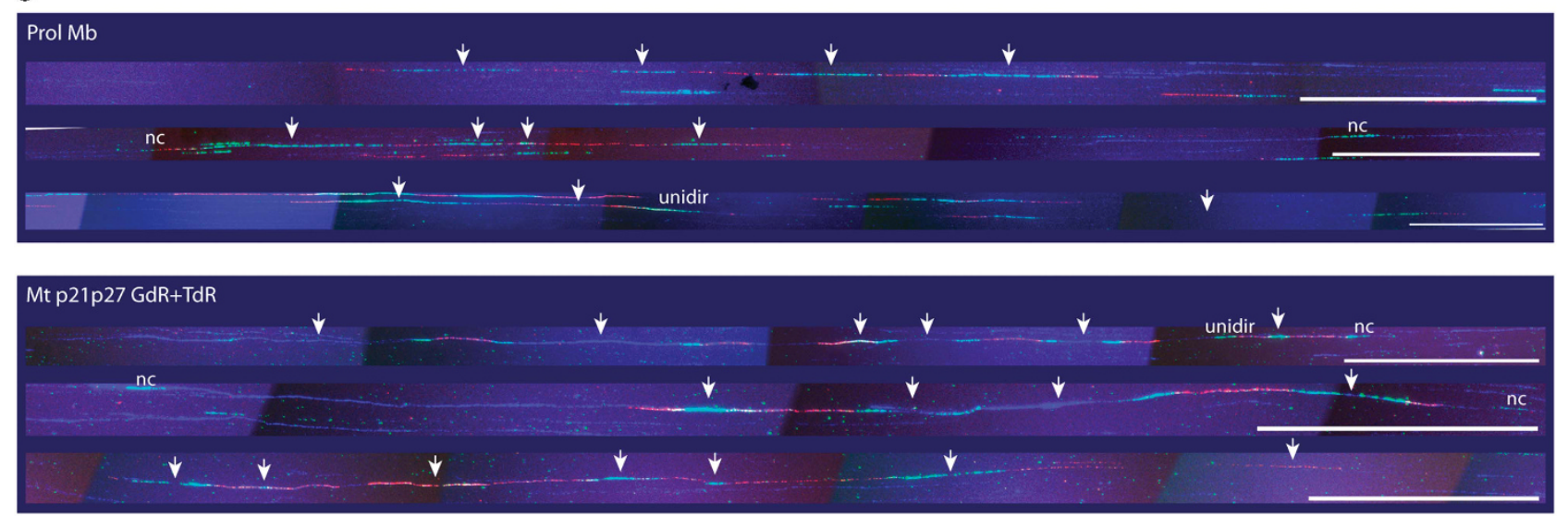

Figure 3 Molecular combing shows that low dNTP levels deeply affect fork rates in reactivated myotubes. (a) Fork rate distributions evaluated by molecular combing in proliferating myoblasts and in myotubes reactivated as indicated. The samples are highly statistically different $(P<0.001$, Kruskal-Wallis non-parametric test). (b) Representative replication forks for the three samples evaluated. Images are not in the same scale, due to the large differences in fork rates (calibration bar $=100 \mathrm{~kb}$ ). Green and red fluorescent tracks correspond to the first and second pulse (IdU and CldU, respectively), whose duration was modulated according to the average replication fork rate ( $30 \mathrm{~min}+30 \mathrm{~min}$ for Prol $\mathrm{Mb}$ and Mt p21p27i GdR+TdR; $60 \mathrm{~min}+60 \mathrm{~min}$ for Mt p21p27i). DNA is counterstained by immunodetection (blue fluorescence). Black arrows indicate origin positions. To appreciate fork rate differences among the three samples, the ideogram of a replication fork running at $1 \mathrm{~kb} / \mathrm{min}$ is drawn above each microscope image. (c) Inter-origin distance distributions in proliferating myoblasts and rMt. The samples are highly statistically different $(P<0.001$, Kruskal-Wallis non-parametric test). (d) Length distributions of replication clusters in proliferating myoblasts and rMt. The samples are highly statistically different $(P<0.001$, Kruskal-Wallis non-parametric test). (e) Representative replicating molecules from myoblasts and reactivated myotubes. Fluorescence as described for panel (b). White arrows indicate the positions of bidirectional replication origins. unidir, unidirectional forks; nc, not classified. Calibration bar $=200 \mathrm{~kb}$

To assess whether the low levels of TK1 contribute to dNTP pool synthesis and DNA replication in $\mathrm{rMt}$, we silenced TK1 in myotubes reactivated by CKI-depletion (Figure 6). In the absence of suitable antibodies against the murine enzyme, we resorted to a functional assay to demonstrate the efficacy of TK1 knockdown. Figure $6 \mathrm{a}$ shows that 5-ethynyl-2'deoxyuridine (EdU) incorporation into $r M t$ largely depends on TK1 expression, as low as it might be in these cells. Myotubes were similarly reactivated by CKI suppression, independently of TK1 interference, as shown by the expression of the cell cycle-dependent protein MCM7. However, in
TK1-knockdown cells, EdU incorporation was nearly undetectable, as its conversion into EdUTP was severely impaired. TK1 knockdown markedly reduced DNA replication in myotubes reactivated by CKI RNAi (Figure $6 b$ and Table 1), confirming that TK1 activity, albeit low (Figure 5a), was limiting for DNA synthesis in rMt. In contrast, suppression of DCTD, which concurs to convert deoxycytidine monophosphate into thymidine monophosphate (see later, Figure 8b), had no effect on DNA replication (Table 1).

Similar experiments were carried out with myotubes reactivated by E1A expression. In these cells, TK1 RNAi 
a

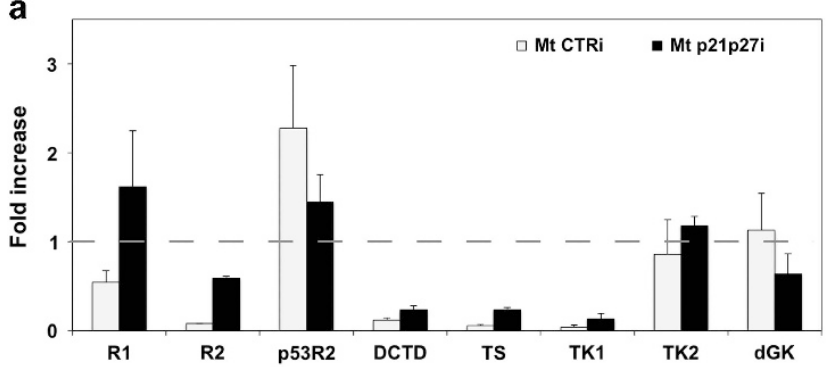

b

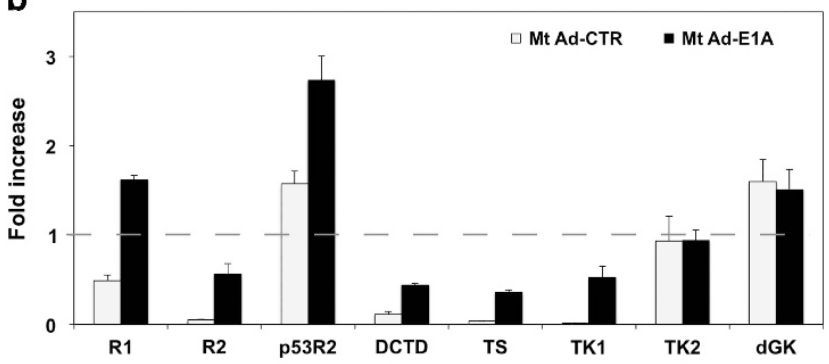

C

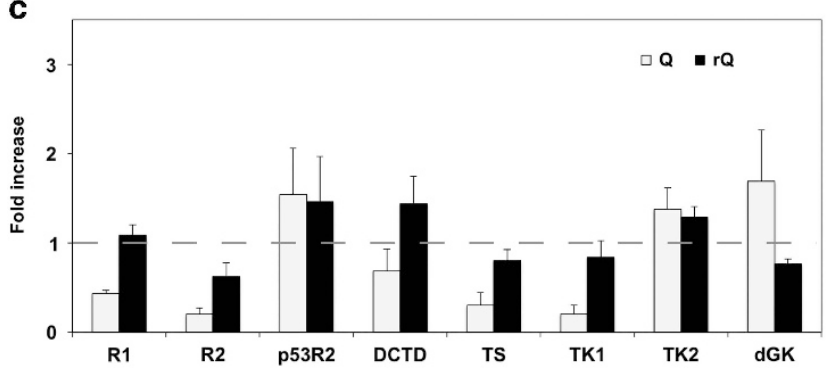

Figure 4 Thymidine synthetic enzymes are not upregulated in reactivated myotubes. (a) Myotubes were reactivated by p21 and p27 depletion (Mt p21p27i) or (b) E1A expression (Mt Ad-E1A). The mRNA levels of several dNTP synthetic enzymes were quantitated by QPCR in myotubes treated as indicated, proliferating myoblasts, and (c) quiescent myoblasts $(Q)$ or myoblasts reactivated after quiescence $(\mathrm{rQ})$. In all cases, reactivated cells were harvested in the midst of $S$ phase. Histograms show fold increases in comparison with proliferating myoblasts, made equal to 1 (dashed lines) (averages \pm S.E.M. of two independent experiments). $\mathrm{R} 1, \mathrm{R} 2$, and p53R2 indicate the corresponding ribonucleotide reductase subunits; DCTD, deoxycytidine monophosphate deaminase; dGK, deoxyguanosine kinase; TK1 and TK2, thymidine kinases 1 and 2; TS, thymidylate synthase

reduced DNA replication, though less markedly than in CKIsuppressed cells (Table 1). Presumably, this result reflects the upregulation of other dNTP synthetic enzymes in E1Areactivated myotubes (Figure 4 ). Indeed, in these cells, suppression of DCTD did reduce DNA replication (Table 1). In agreement, double suppression of TK1 and DCTD resulted in an even stronger limitation of DNA synthesis.

Altogether, these results show that the low expression of enzymes involved in dTTP anabolism is responsible for low DNA precursor levels and confirm that expanding the dNTP pool increases DNA synthesis.

The TK1 gene is deeply downregulated in myotubes and unresponsive to cell cycle re-entry. TK1 is a cell cycledependent gene, whose transcription is strongly downregulated in non-proliferating cells and sharply increases at the $G_{1} / S$
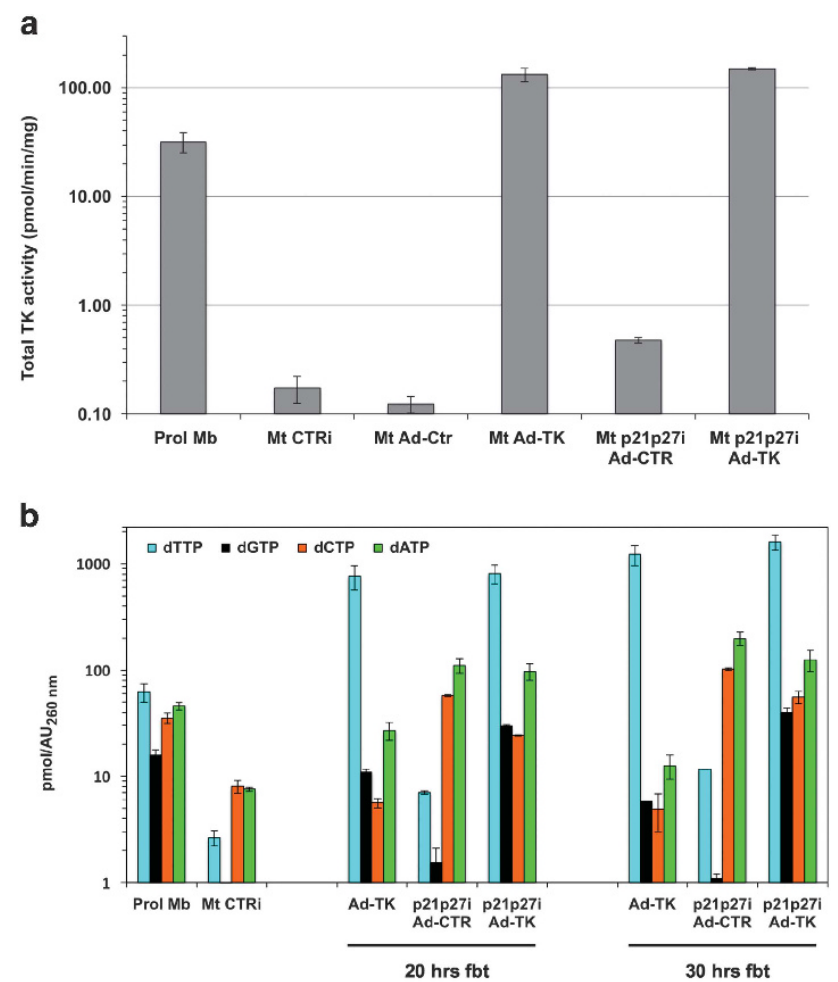

C

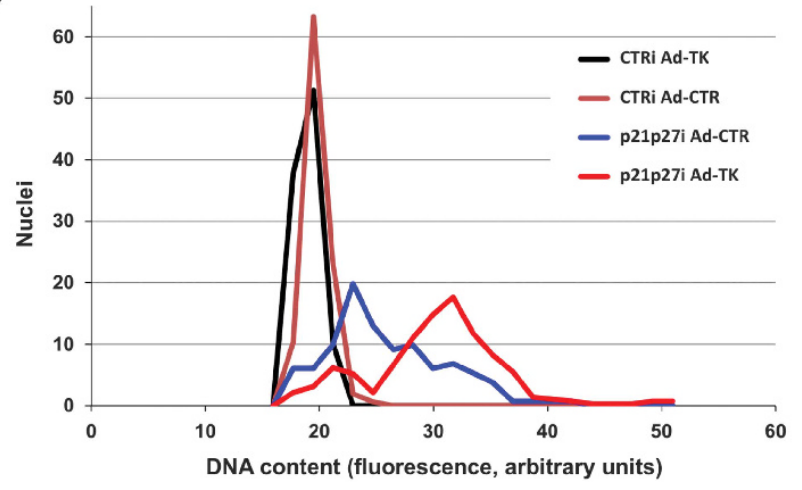

Figure 5 Exogenous thymidine kinase expression increases dTTP and dGTP levels and extends DNA synthesis in reactivated myotubes. (a) Total TK activity in myoblasts or myotubes, treated as indicated and collected $30 \mathrm{~h}$ from the beginning of treatment (fbt). The scale is logarithmic to accommodate widely different values. (b) dNTP pools measured at the indicated times in myoblasts or myotubes treated as shown. Logarithmic scale. (c) Microfluorimetric measurements of DNA content in the indicated cell samples, harvested $30 \mathrm{~h}$ fbt. The graph refers to one of the two experiments; Supplementary Table $\mathrm{S} 6$ summarizes all the results. Ad-CTR, infection with control adenovirus; Ad-TK, infection with the adenovirus expressing herpes simplex virus TK; CTRi, interference with control siRNA

boundary upon cell cycle re-entry. ${ }^{27,28}$ Myotubes, however reactivated, upregulate most cell cycle-related genes (Camarda et al., ${ }^{29}$ Figures $4 \mathrm{a}$ and $\mathrm{b}$; and our unpublished global gene expression data). Thus it is remarkable that, in CKI-depleted rMt, the expression of TK1 and a few other dNTP synthetic enzymes is held tightly down.

To explore the causes of this distinct behavior, we investigated the epigenetic regulation of TK1 and, as controls, some other genes differentially regulated in myoblasts and myotubes. Figure $7 a$ shows that Cyclin $A 2$ is silenced in 
a
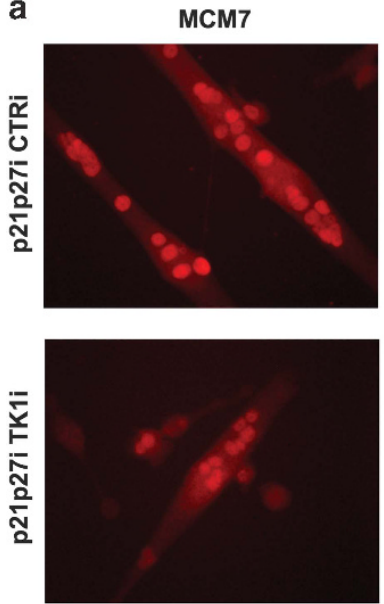
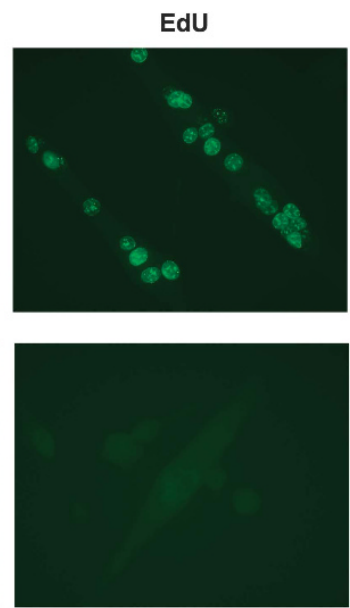
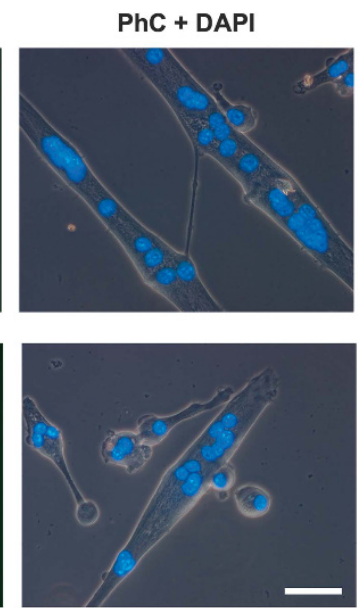

b

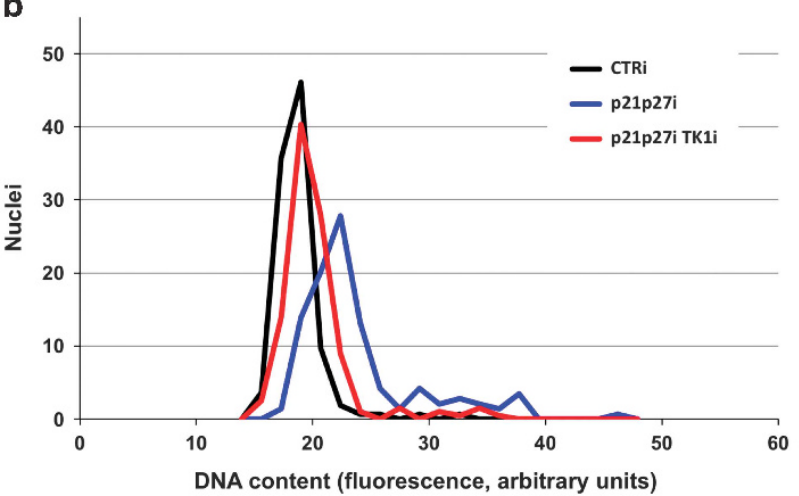

Figure 6 Suppression of TK1 impairs DNA replication in reactivated myotubes. (a) Double immunofluorescence for MCM7 and EdU in p21p27-depleted myotubes, additionally subjected to interference with TK1 or control siRNA. Cultures were fixed during S phase and stained as indicated. PhC, phase contrast. Bar, $50 \mu \mathrm{m}$. (b) Microfluorimetric measurements of DNA content in the indicated cell samples, assayed $31 \mathrm{~h}$ after the beginning of treatment

Table 1 DNA replication in reactivated myotubes: role of TK1 and DCTD

\begin{tabular}{|c|c|c|c|c|c|c|}
\hline RNAi & CTR & p21p27, CTR & p21p27, TK1 & p21p27, DCTD & & Comparison \\
\hline $\begin{array}{l}\text { DNA content }(\% \text { increase }>2 n) \\
P(\text { Kolmogorov-Smirnov) }\end{array}$ & 0 & 26.6 & $\begin{array}{l}7.6 \\
<0.000001\end{array}$ & $\begin{array}{l}31.5 \\
\mathrm{NS}\end{array}$ & & p21p27, CTR \\
\hline $\begin{array}{l}\text { Infection } \\
\text { RNAi }\end{array}$ & $\begin{array}{l}\text { Ad-CTR } \\
-\end{array}$ & $\begin{array}{l}\text { Ad-E1A } \\
\text { CTR }\end{array}$ & $\begin{array}{l}\text { Ad-E1A } \\
\text { TK1 }\end{array}$ & $\begin{array}{l}\text { Ad-E1A } \\
\text { DCTD }\end{array}$ & $\begin{array}{c}\text { Ad-E1A } \\
\text { TK1, DCTD }\end{array}$ & \\
\hline $\begin{array}{l}\text { DNA content }(\% \text { increase }>2 n) \\
P(\text { Kolmogorov-Smirnov) }\end{array}$ & 0 & 43.8 & $\begin{array}{l}27.5 \\
<0.000001\end{array}$ & $\begin{array}{l}36.3 \\
<0.0054\end{array}$ & $\begin{array}{l}16.6 \\
<0.000001\end{array}$ & Ad-E1A, CTR \\
\hline
\end{tabular}

Abbreviation: NS, not significant

postmitotic myotubes but is upregulated in rMt, consistent with its crucial role in the cell cycle. In contrast, Pax7, a musclespecification transcription factor, is expressed in myoblasts, silenced in myotubes, and not reactivated in rMt. Finally, the muscle differentiation factor Myogenin is not expressed in myoblasts but is present in myotubes, irrespective of their cell cycle status.

Chromatin immunoprecipitations (ChIPs) were carried out for acetylated lysine 9 of histone H3 (H3K9Ac), a marker associated with actively transcribed chromatin. ${ }^{30}$ H3K9Ac enrichment at gene promoters closely reflected the expression of each gene in the various cell types and conditions (Figure 7b). In particular, it was highly enriched at the TK1 promoter in myoblasts, but scarcely so in myotubes, and even less present in rMt. This result indicates that the TK1 regulatory region is transcriptionally active in myoblasts but adopts a repressed state in myotubes. Different from Cyclin $\mathrm{A} 2$, whose promoter is reactivated upon re-entry into the cell cycle (Figure $7 b$ ), the TK1 gene remains inactive even after myotube reactivation.

Unlike that of TK1, the expression of the RNR subunit R2 increases in response to myotube reactivation. Indeed, H3K9Ac, though markedly reduced at the R2 promoter in myotubes compared with myoblasts, promptly raises upon cell cycle re-entry (Supplementary Figure S3A), underscoring the differential regulation of these key metabolic enzymes. 
a

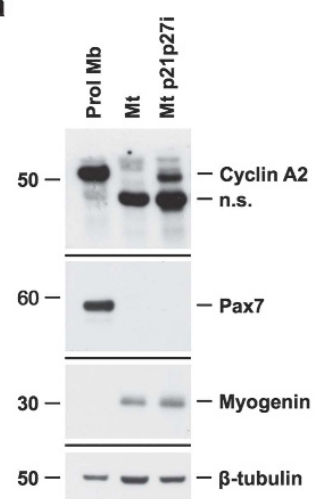

b

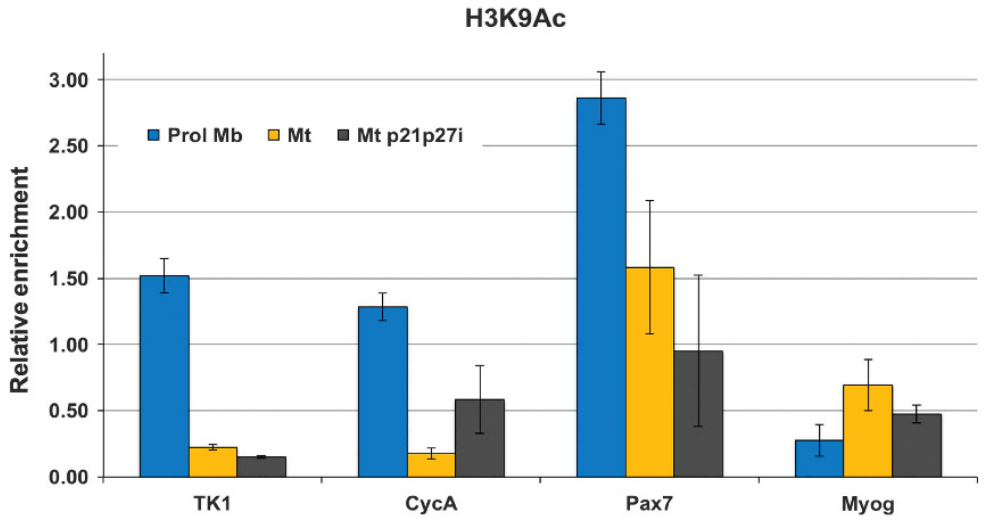

C

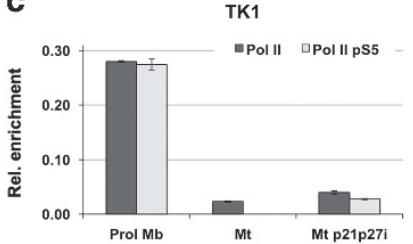

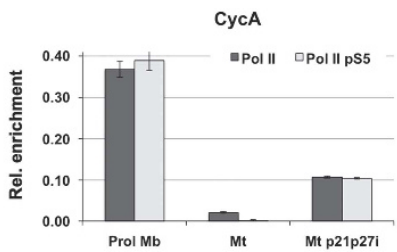
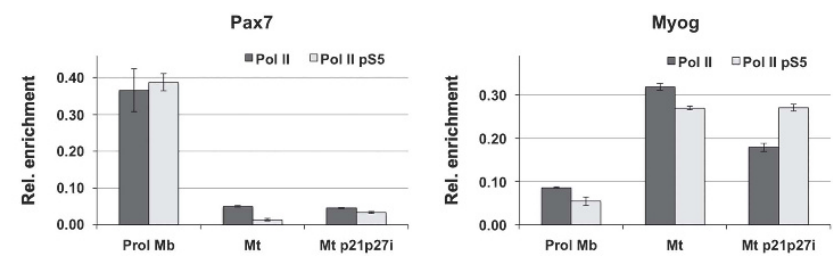

Figure 7 The TK1 locus is strongly repressed in terminally differentiated myotubes and is not reactivated by cell cycle re-entry. (a) Western blotting analyses of the indicated proteins in the cells shown. Reactivated myotubes were harvested in middle $S$ phase. $\beta$-Tubulin is the loading control. n.s., non-specific band. (b) ChIP-measured enrichment in H3K9AC at the indicated promoters in the cells shown. rMt were collected in middle $S$ phase (average \pm S.E.M. of $\geq 2$ independent experiments). (c) ChIP-measured enrichment in total RNA polymerase II (Pol II) and active Pol II (Pol II pS5) at the same promoters as in panel (b) (average \pm S.E.M. of three replicas)

ChIPs for total RNA polymerase II and for its active form, phosphorylated on CTD-repeat Ser $5,{ }^{31}$ closely paralleled the above results (Figure 7c and Supplementary Figure S3B), indicating substantial transcription only from promoters heavily marked with $\mathrm{H} 3 \mathrm{~K} 9 \mathrm{Ac}$.

These data indicate that TK1 is a rare cell cycle-regulated gene permanently downregulated during skeletal muscle differentiation and mechanistically explain the inability of myotubes to properly expand the dNTP pool in response to cell cycle reactivation.

\section{Discussion}

This paper investigates the causes of incomplete DNA synthesis in cell cycle-reactivated myotubes. ${ }^{11}$ We show that the dNTP pool of rMt is highly imbalanced and limits DNA synthesis. The structure of the enzyme network regulating dNTP concentrations ${ }^{13}$ is altered. Although cell-cycle reactivation restores the S-phase-specific transcription of RNR, insufficient expression of genes encoding key dTMP synthetic enzymes, notably TK1, drastically hampers the production of dTTP (Figure 8a).

The demonstration that some genes required for cell proliferation, different from most others, are permanently downregulated in myotubes (Camarda et al., ${ }^{29}$ Figures $4 a$ and $\mathrm{b}$; and our unpublished data) explains in part the inability of these cells to fully replicate DNA upon cell cycle reactivation. This mechanism might constitute a safeguard against unwarranted mitotic activity and explains in part the extreme difficulties encountered in inducing terminally differentiated muscle cells to proliferate.
DNA combing analyses have shown that the preponderant DNA duplication defect in $\mathrm{rMt}$ is a dramatic $85 \%$ reduction in replication fork rate (Figure 3a and Supplementary Table S4). The most parsimonious interpretation of this result is that such low replication speed is caused by an inadequate supply of deoxynucleotides. Restoration of sufficient dNTP levels nearly triples fork speed, which nonetheless remains significantly reduced, compared with that of myoblasts. These findings further illustrate the need to search for other mechanisms that impede myotube proliferation.

In rMt, DNA replication is limited by low levels of dTTP and possibly dGTP. Indeed, any experimental intervention that raises both pools results in extended DNA synthesis, whereas increasing solely dGTP is ineffective. When cells are incubated with TdR, the concentrations of dGTP and dTTP rise jointly, as dTTP induces GDP reduction by $\mathrm{RNR}^{26}$ The pathway converting dCMP to dTMP (Figure $8 b$ ) is virtually inactive in myotubes, as shown by the observation that DCTD knockdown does not affect DNA replication in rMt (Table 1). Such inactivity causes accumulation of dCTP and lack of dTTP, which is not available for its twofold function as DNA precursor and allosteric stimulator of GDP reduction.

TK1, albeit limiting in rMt, has a crucial role in dTTP synthesis, as demonstrated by the reduction in DNA replication caused by its silencing (Table 1). TK1 and other genes involved in dTTP synthesis are upregulated somewhat more in myotubes reactivated by E1A expression (Figure $4 b$ ) than in CKI-depleted ones. Accordingly, the former duplicate their DNA more extensively (Figure $2 \mathrm{e}$ ), probably reflecting the fact that adenovirus E1A has evolved as an efficient S-phase inducer, to allow viral replication in host cells. 


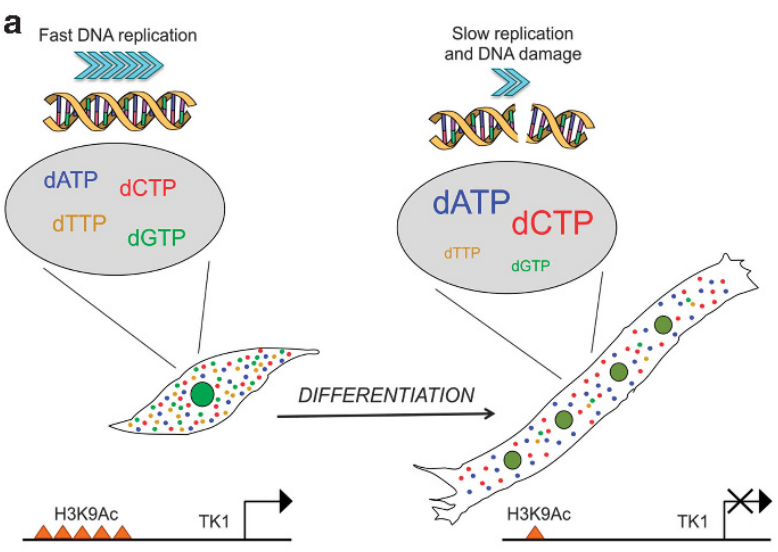

Myoblasts

Myotubes

b

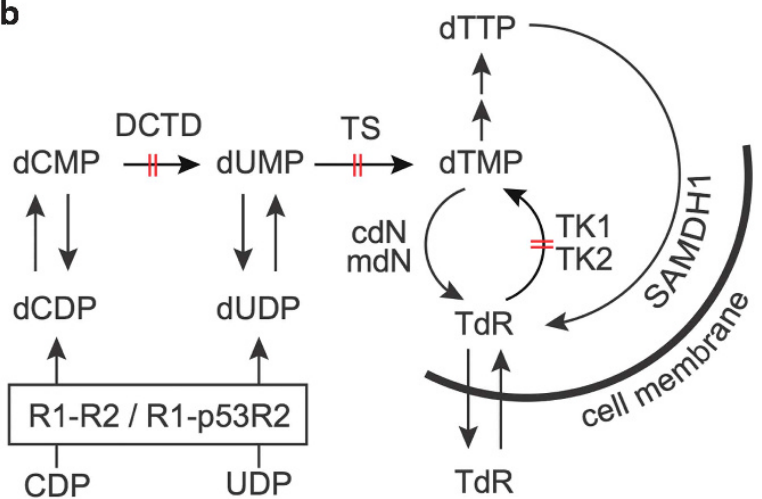

Figure 8 Graphic summary. (a) Graphical representation of the main results of this work. The nucleotide pool is adequate and balanced in myoblasts, allowing fast DNA replication. Upon differentiation into myotubes, muscle cells deeply silence transcription of a few genes involved in dTTP synthesis, exemplified by TK1. Accordingly, such genes display markedly reduced histone $\mathrm{H} 3$ lysine 9 acetylation and their products are depleted in myotubes, in spite of cell cycle re-entry. Loss of key metabolic enzymes leads to a dramatic fall in dTTP and, indirectly, dGTP levels. As a consequence, cell cycle reactivation in myotubes is marred by slow DNA replication, accompanied by DNA damage. (b) Synthesis of thymidine triphosphate in mammalian cells. dTTP synthesis occurs through two pathways: de novo synthesis by RNR and salvage of TdR. The latter is initiated by two thymidine kinases, cytosolic TK1, expressed in S phase, and mitochondrial TK2, which is a constitutively expressed enzyme. During S phase, RNR is composed by homodimers of the R1 and $\mathrm{R} 2$ subunits, whereas in non-dividing or differentiated cells $\mathrm{R} 2$ is replaced by p53R2. De novo-produced thymidine monophosphate (dTMP) derives from deoxyuridine monophosphate (dUMP) via methylation by TS. In its turn, dUMP derives from (i) dephosphorylation of dUDP produced by RNR and (ii) deamination of deoxycytidine monophosphate (dCMP) by DCTD. The latter process prevails in mammalian cells. ${ }^{41}$ The salvage pathway receives TdR from the intracellular catabolism of dTMP by 5 '-nucleotidases (cytosolic cdN and mitochondrial mdN) and of dTTP by the nuclear dNTP-triphosphohydrolase SAMHD1 or via uptake from the extracellular environment. In terminally differentiated myotubes DCTD, TS and TK1 are strongly repressed (barred arrows) and reactivation by p21 and p27 silencing fails to induce them to levels approaching those of proliferating cells. Thus both pathways converging on dTMP, an essential precursor of dTTP, are severely impaired in reactivated myotubes

Our results show that the dTTP synthetic pathways are controlled in a selective and especially stringent manner in postmitotic muscle cells. It is worth noting that herpes simplex virus type I, whose natural host is the neuron, carries its own ribonucleotide reductase- and TK-encoding genes. ${ }^{32}$ This suggests that persistent TK silencing is a feature that characterizes other terminally differentiated cell types, besides myotubes.

To conclude, we propose a model whereby DNA synthesis in rMt is severely limited by the lack of DNA precursors, resulting from the coordinated, reactivation-resistant silencing of genes involved in dTTP synthesis. This unexpectedly tight control might serve to prevent undue proliferative activity in myotubes. In this respect, we note that at least one natural condition induces re-entry into the cell cycle of muscle nuclei, namely infection by Trichinella spiralis. ${ }^{33}$

\section{Materials and Methods}

Cells and adenoviruses. Muscle satellite cells were isolated following a previously described protocol, ${ }^{34}$ with minor modifications. Briefly, hindlimbs were removed from 3-week-old mice, and the muscle mass was minced using scissors. Cells were enzymatically dissociated by a $30 \mathrm{~min}$ incubation in $0.1 \mathrm{mg} / \mathrm{ml}$ collagenase/dispase solution (Roche, Basel, Switzerland), followed by a $45 \mathrm{~min}$ digestion in $1 \mathrm{mg} / \mathrm{ml}$ collagenase solution (Sigma Aldrich, St. Louis, MO, USA), pipetting several times. When tissue suspension no longer showed visible clumps, the homogenate was filtered through a $70 \mu \mathrm{m}$ nylon mesh cell strainer (Becton Dickinson, East Rutherford, NJ, USA). Cells were collected by centrifugation and plated on gelatin-coated dishes in growth medium (GM): Ham's F-10 medium (Life Technologies, Carlsbad, CA, USA) supplemented with $20 \%$ fetal bovine serum (FBS), $3 \%$ chicken embryo extract, and $2.5 \mathrm{ng} / \mathrm{ml}$ basic-FGF. Spontaneous immortalization occurred within 10 passages. Differentiation was induced by plating cells on gelatin-coated dishes in differentiation medium: Dulbecco's Modified Eagle Medium (Life Technologies) supplemented with $10 \%$ FBS. After $48 \mathrm{~h}$, the vast majority of the cells were differentiated (differentiation index $>95 \%$ ).

Satellite cells were made quiescent by culturing them in suspension on semisolid medium (GM with $0.7 \%$ Noble Agar (Life Technologies) for $21 \mathrm{~h}$. Cell cycle re-entry was triggered by replating them on gelatin-coated dishes in liquid GM.

E1A was expressed using the d1520 mutant adenovirus previously described. ${ }^{5}$ This virus was used at a multiplicity of infection (MOI) of 350 . The recombinant adenovirus expressing herpesvirus thymidine kinase was kindly donated by Dr. Rafael Aldabe (CIMA, University of Navarra, Navarra, Spain). This virus was used at an MOI 300. When both infection and RNAi were performed, the latter was achieved $1 \mathrm{~h}$ after the infection.

Gene silencing by siRNA transfection. Myotubes were transfected with the HiPerFect transfection reagent (Qiagen, Hilden, Germany) combined with siRNA. siGenome duplex (Dharmacon, Lafayette, CO, USA) siRNAs were used to interfere with murine CDKN1A (p21) and CDKN1B (p27) transcripts. The antisense sequences for p21 and p27 were 5'-P.GAACAGGUCGGACAUCACCUU-3' and 5'P.UAUCCCGGCAGUGCUUCUCUU-3', respectively. The antisense sequence to mouse SAMHD1 was 5'-AUAGAAGUGAACACGAUCGAT-3' (Qiagen). Interference with murine TK1 and DCTD was carried out using Dharmacon siGenome SMART pools M-042809-00 and M-058063-02, respectively. The siRNA to GFP (antisense sequence: 5'-GGUGCGCUCCUGGACGUAGCCUU-3', Sigma Aldrich) was transfected as a control. Transfection complexes were kept in the culture medium throughout the experiment.

Deoxynucleoside complementation. GdR and TdR (Sigma Aldrich) were added, singularly or in combination, to rMt culture medium at final concentrations of 500 and $100 \mu \mathrm{M}$, respectively, $17 \mathrm{~h}$ after siRNA transfection. GdR and $\mathrm{TdR}$ concentrations were chosen on empirical bases, taking into account the instability of $\mathrm{GdR}$ and the potential cell cycle-blocking effects of high levels of TdR. The concentration ranges considered were $10-100 \mu \mathrm{M}$ for $\mathrm{TdR}$ and $100-500 \mu \mathrm{M}$ for $\mathrm{GdR}$. The concentrations selected were the ones restoring myoblast-like levels of dTTP and dGTP in rMt.

dNTP pool analysis. dNTP pools were extracted from cell cultures with $60 \%$ ice-cold methanol for $1 \mathrm{~h}$. Cells left on the plate were dissolved in $0.3 \mathrm{M} \mathrm{NaOH}$ and the absorbance at $260 \mathrm{~nm}$ of the lysates was used as an index of cell mass, in turn an approximation for cell number. ${ }^{35}$ dNTP pool quantitations were performed by an in vitro-modified DNA polymerase-based assay, as described. ${ }^{16}$ Two different 
aliquots of each pool extract were analyzed and pool sizes were normalized by the A260 $\mathrm{nm}$ of the $\mathrm{NaOH}$ lysates. ${ }^{17}$

Immunofluorescence and western blotting. Immunofluorescence was performed on cells fixed with $4 \%$ formaldehyde and permeabilized with $0.25 \%$ Triton X-100 (Sigma Aldrich). For BrdU staining, DNA was denatured with $2 \mathrm{M}$ hydrochloric acid. Primary mouse monoclonal antibodies were used to detect: BrdU (Bu20a clone, DAKO); MCM7 (DCS-141 clone, Santa Cruz, Dallas, TX, USA); myosin heavy chain $\left((\mathrm{MF} 20)^{36}\right) ; \gamma$-H2AX (JBW301 clone, Millipore, Billerica, MA, USA). Ki67 was detected with a rabbit polyclonal antibody (Abcam, Cambridge, UK, cat. no. AB9260). The modified thymidine analog EdU, incorporated into newly synthesized DNA, was revealed by the Click-iT Imaging Kit (ThermoFisher Scientific, Waltham, MA, USA). AlexaFluor 488- or 594-conjugated secondary antisera to mouse or rabbit IgG (Life Technologies) were used. Nuclei were counterstained with $0.2 \mu \mathrm{g} / \mathrm{ml}$ DAPI for $20 \mathrm{~min}$.

To prepare total cell extracts, cells were harvested, washed in PBS, and lysed with RIPA buffer, containing $500 \mathrm{mM} \mathrm{NaCl}$. Proteins were separated on gradient, 4-12\% polyacrylamide gels and analyzed by western blotting with the following antibodies: anti-SAMHD1 (Abcam, clone 1A1), anti-Cyclin A2 (C-19, Santa Cruz), anti-Pax7 (Developmental Studies Hybridoma Bank, University of lowa, IA, USA, cat. no. pax7, RRID:AB_528428), anti-Myogenin (FD5 hybridoma), anti- $\beta$-tubulin (2-28-33 clone, Sigma Aldrich).

DNA quantitation and $\boldsymbol{\gamma}-\mathrm{H} 2 \mathrm{AX}$ expression. Microfluorimetry was used to quantitate DNA in myotube nuclei stained with DAPI, as multinucleated myotubes are not readily amenable to cytofluorimetry. $r$ Mt were allowed to synthesize DNA until shortly before $\mathrm{M}$ phase or cell death. The cells were then fixed, immunostained for MCM7 (a cell cycle-dependent marker of reactivation), and counterstained with DAPI. ${ }^{37}$ To assess the extent of DNA replication in rMt and exclude non-reactivated ones, DAPI fluorescence intensity was measured solely in MCM7-positive nuclei. Control myotubes were subjected to the same treatments, but DAPI microfluorimetry was performed on all nuclei, which were uniformly MCM7-negative. Random myotube images were captured at room temperature, using an Axioskop 2 Plus fluorescence microscope (Carl Zeiss, Oberkochen, Germany) equipped with a Neofluar $40 \times$ objective (NA 0.75) and an AxioCam camera (Carl Zeiss). Images were captured with the ZEN 2012 software (Carl Zeiss). The images were analyzed with the public domain software ImageJ (v. 1.43u). DAPI fluorescence intensity (proportional to DNA content) was measured in all well-defined individual nuclei in the captured images, with local background subtraction. Fluorescence values, in arbitrary units, were distributed into 20 , equally wide intensity channels and the percentages of total nuclei falling into each channel were plotted. The curves thus obtained were integrated to calculate the average fluorescence intensity (AFI) of the sample. In each experiment, the DNA content of the test nuclei was expressed as a percentage of increase of their AFI over AFI of control nuclei ( $2 n$, non-reactivated myotubes). $\gamma$-H2AX levels were similarly measured, except that reactivated nuclei were detected by Ki67 immunofluorescence.

DNA combing assay. Exponentially growing myoblasts and rMt were subjected to sequential pulses with two deoxythymidine analogs: 5-iodo-2'-deoxyuridine (IdU, Sigma Aldrich) and 5-Chloro-2'-deoxyuridine (CldU, Sigma Aldrich), both at $100 \mu \mathrm{M}$ final concentration. The length of the pulses were: $30 \mathrm{~min}$ each for myoblasts and deoxynucleoside-supplemented $\mathrm{rMt}$; $60 \mathrm{~min}$ each for control rMt. Immediately afterwards, cells were collected and immobilized into agarose plugs (100-200 000 cells/plug). Plugs were then incubated overnight in $2 \mathrm{mg} / \mathrm{ml}$ proteinase $\mathrm{K}$ solution (1\% N-laurosylsarcosine, 0.1 M EDTA pH 8, 0.01 M Tris-HCl pH 8, 0.02 M NaCl) and DNA was released by incubation with three units of $\beta$-agarase (New England Biolabs, Ipswich, MA, USA) in 0.1 M MES, pH 6.1. DNA stretching on silanised surfaces was performed according to a validated procedure. ${ }^{21,24}$ Preparations were denatured in $1 \mathrm{M} \mathrm{NaCl}$ and $50 \mathrm{mM} \mathrm{NaOH}$ for $15 \mathrm{~min}$ before being incubated for $30 \mathrm{~min}$ with antiBrdU antibodies, cross-reacting, respectively, with IdU (Becton Dickinson, developed in mouse, 2:7 dilution) and CldU (Abcam, developed in rat, 1:40 dilution). Slides were then incubated for $30 \mathrm{~min}$ with a mixture of AlexaFluor 488-conjugated anti-mouse IgG and AlexaFluor 594-conjugated anti-rat IgG (all from Life Technologies, 1:50 dilution). DNA was immunodetected by three successive incubations steps, using, respectively, primary mouse IgG2A anti-ssDNA (Millipore, Clone 3034, 1:25 dilution, $90 \mathrm{~min})$, AlexaFluor 350-conjugated anti-mouse IgG2A developed in goat, and AlexaFluor 350-conjugated anti goat lgG developed in donkey (both secondary antibodies from Life Technologies, 1:50 dilution, $30 \mathrm{~min}$ ). In summary, DNA was counterstained with blue fluorescence and replication forks are detected by green and red fluorescences. A motorized fluorescence microscope (Zeiss Axio Imager.M1) equipped with a CCD camera (Photometrix, Kew, Victoria, Australia, Coolsnap $\mathrm{HQ}^{2}$ ) was used to score slides under a $40 \times$ oil immersion objective $(N A=1.30)$. Adjacent fields were acquired with the necessary filter sets. By aligning merged images (using Adobe (San Jose, CA, USA) Photoshop CS2), whole DNA molecules were reconstructed. Fluorescent patterns were measured by the Metavue Research Imaging System (Molecular Devices, Sunnyvale, CA, USA), according to the molecular combing calibration factor $(1 \mu \mathrm{m}=2 \mathrm{~kb})$ and the magnification provided by objective and CCD camera $(1$ pixel $=0.16125 \mu \mathrm{m})$. The lengths of replication tracks and their spatial organization define the replication profile (fork rate, inter-origin distances, initiation sites, size of the replication clusters). Stringent criteria were applied to interpret the fluorescent patterns. A detailed description can be found in Palumbo et al. ${ }^{24}$

Gene expression analysis by qPCR. Total RNA was isolated from cell cultures with TRIzol reagent (ThermoFisher Scientific) and CDNA was synthesized from $2 \mu \mathrm{g}$ of RNA using the High Capaciy cDNA Reverse Transcription Kit (Applied Biosystems, Foster City, CA, USA), according to the manufacturer's instructions. qPCR was performed with $0.3 \mu \mathrm{l}$ of cDNA (from a 20- $\mu$ l total reaction volume), using SYBR Green cyanine dye (SensiMix, Bioline, Taunton, MA, USA), on a 7500 Fast Real-Time PCR System (Applied Biosystems). The specificity of qPCR reactions was verified by the melting temperature method. Each gene was assayed in triplicate and the mean threshold cycle $(\mathrm{Ct})$ value was calculated. The Pctk1 housekeeping gene (hg), found to be homogeneously expressed in all samples, was used to normalize target gene $(\mathrm{tg})$ results. Proliferating myoblasts were used as reference $\left(\mathrm{Ct}_{\mathrm{ref}}\right)$. Gene expression fold-change was calculated as $2^{-\Delta \Delta \mathrm{Ct}}$, where $\Delta \Delta \mathrm{Ct}=\left(\mathrm{Ct}_{\mathrm{tg}}-\mathrm{C}_{\mathrm{hg}}\right)-\left(\mathrm{Ct}_{\mathrm{ref}}-\mathrm{Ct}_{\mathrm{hg}}\right)$, setting to 1 the expression of tg in proliferating myoblasts. The following primers were used: RNR-R1 (5'-CCCAATGAGTGT CCTGGTCT-3', 5'-GTTCTGCTGGTTGCTCTTCC-3'); RNR-R2 (5'-CCTACTAA CCCCAGCGTTGA-3', 5'-GTTTCAGAGCTTCCCAGTGC-3'); RNR-p53R2 (5'ACACACGCACACACCACCTGTA-3', 5'-TGACAAATGGGAAGCACAGAGC-3'); DCTD (5'-GAAGCAAGGACCCAAGTTTCC-3', 5'-CCTTCACATCAGCCGAGTTCT -3'); TS (5'-ATGTGGTGAATGGGGAACTGT-3', 5'-GAGCTTTGGGAAAGGTCTT GG-3'); TK1 (5'-CGATGTGACCCAGGAGTCC-3', 5'-GGAGAGTGTGGTGAAGCT CA-3'); TK2 (5'-TGTCCTTGTGTGAGAGAGCAGT-3', 5'-CTCCAGGGTATACGGT CATCAT-3'); dGK (QuantiTect primer assay, Qiagen); and Pctk1 (5'-ACT GGAGACCTACATCAAGC-3', 5'-GGATAGCAGTACAGGGTG-3').

Thymidine kinase enzymatic assay. Cultures of exponentially growing myoblasts and myotubes were rinsed three times with ice-cold $0.9 \% \mathrm{NaCl}$, drained, and collected by scraping in ice-cold lysis buffer $(10 \mathrm{mM}$ Tri-HCl, pH 7.5, 0.5\% Triton $\mathrm{X}-100,2 \mathrm{mM}$ EDTA, $1 \mathrm{mM}$ DTT), supplemented with $0.2 \mathrm{M} \mathrm{NaCl}$ and protease inhibitors. Lysates were then centrifuged $\left(16000 \times \mathrm{g}\right.$ at $4^{\circ} \mathrm{C}$ for $\left.30 \mathrm{~min}\right)$ and the supernatant was aliquoted and stored at $-80^{\circ} \mathrm{C}$. Protein concentration was determined by the Bradford colorimetric assay. TK activity was assayed as described in Franzolin et al..$^{38}$ The substrate was $1 \mu \mathrm{M}\left[{ }^{3} \mathrm{H}\right] \mathrm{TdR}$ (Perkin-Elmer Life Sciences, Waltham, MA, USA) $(800 \mathrm{cpm} / \mathrm{pmol})$ and two different aliquots of extracts were used to check for proportionality of the assay. We expressed TK activity as pmol product/min/ mg protein.

Chromatin immunoprecipitation. ChIP experiments were performed as previously described. ${ }^{39}$ In particular, crosslinking was performed by treating cell cultures with $1 \%$ formaldehyde, for $10 \mathrm{~min}$, at $22^{\circ} \mathrm{C}$; the reaction was stopped by adding glycine to a final concentration of $0.125 \mathrm{M}$. Cells were then washed, collected by scraping in PBS, centrifuged, and lysing the cytoplasms with ChIP lysis buffer (5 mM Pipes, $85 \mathrm{mM} \mathrm{KCl,} \mathrm{0.5 \%} \mathrm{NP-40).} \mathrm{The} \mathrm{nuclei} \mathrm{thus} \mathrm{isolated} \mathrm{were}$ resuspended in ChIP sonication buffer (1\% SDS, $10 \mathrm{mM}$ EDTA, $50 \mathrm{mM}$ Tris $\mathrm{HCl}, \mathrm{pH}$ 8) and optimal chromatin fragmentation (size: $0.3-1 \mathrm{~Kb}$ ) was empirically obtained. Chromatin (30-50 $\mu \mathrm{g} / \mathrm{ChIP})$ was then diluted in ChIP dilution buffer $(0.01 \%$ SDS, $1.2 \mathrm{mM}$ EDTA, $16.7 \mathrm{mM}$ Tris $\mathrm{HCl}$, pH8, 1.1\% Triton X-100, $167 \mathrm{mM} \mathrm{NaCl}$ ), precleared with Protein A/G Plus Agarose beads (Pierce, Waltham, MA, USA), and incubated for $3 \mathrm{~h}$ with Protein A/G Plus Agarose/antibody beads. ChIP buffers were all supplemented with complete protease inhibitors (Roche). The immunoprecipitated chromatin was then washed seven times with buffer A $(0.1 \%$ SDS, $2 \mathrm{mM}$ EDTA, $20 \mathrm{mM}$ Tris $\mathrm{HCl}, \mathrm{pH} 8,1 \%$ Triton X-100, $150 \mathrm{mM} \mathrm{NaCl}$ ) and seven times with buffer $B$ (same as buffer $A$, but with $500 \mathrm{mM} \mathrm{NaCl}$ ). To increase specificity, one wash with buffer C (1 mM EDTA, $10 \mathrm{mM}$ Tris HCl, pH8, $250 \mathrm{mM} \mathrm{LiCl,} 1 \% \mathrm{NP}-40$, $1 \% \mathrm{Na}$-deoxycholate) was performed. DNA fragments were then eluted by incubating the beads with ChIP elution buffer ( $\left.1 \% \mathrm{SDS}, 100 \mathrm{mM} \mathrm{NaHCO}_{3}\right)$ twice for $15 \mathrm{~min}$. Crosslink reversion, proteinase $\mathrm{K}$ digestion, and DNA precipitation were 
performed according to a previously described protocol. ${ }^{39}$ Input DNAs was subjected to nonspecific-lgG ChIP. The following rabbit polyclonal antibodies were used at $4 \mu \mathrm{g} / \mathrm{ChIP}$ : H3K9ac (Millipore cat. no. 06-942); RNA polymerase II (N-20, Santa Cruz); RNA polymerase II phosphorylated on CTD-Ser5 (ab5131, Abcam); non-immune rabbit IgG (Bioscience, East Rutherford, NJ, USA). qPCR was performed on immunoprecipitated DNA fragments as described above and the comparative threshold $(\Delta \mathrm{Ct})$ method was used to determine the relative proportions of specifically immunoprecipitated fragments in different samples. ${ }^{40}$ Briefly, the $\Delta \mathrm{Ct}$ value was calculated for each sample (S) and for control IgG (C) by subtracting the $\mathrm{Ct}$ value of $\mathrm{S}$ or $\mathrm{C}$ from the $\mathrm{Ct}$ value of the input (In). Relative enrichment was then determined as follows: $2^{\Delta \mathrm{Ct}(\mathrm{S})}-2^{\Delta \mathrm{Ct}(\mathrm{C})}$, where $\Delta \mathrm{Ct}(\mathrm{S})=\mathrm{Ct}(\mathrm{In})-\mathrm{Ct}(\mathrm{S})$ and $\Delta \mathrm{Ct}$ $(\mathrm{C})=\mathrm{Ct}(\mathrm{In})-\mathrm{Ct}(\mathrm{C})$. SYBR melting temperature curve and gel electrophoretic analyses were performed to verify the specificity of each qPCR primer couple. For each gene, primer sequences were designed to target the promoter near the transcription start site. The following primers were used: Cyclin A2 $\left(5^{\prime}\right.$ ACTCCGGACGCCCTCATT-3', 5'-AGTTCAAGTAGCCCGCGACTAT-3'); Myogenin (5'-TCACATGTAATCCACTGGAAACGT-3', 5'-AAACCTGAGCCCCCCTCTAA-3'); PAX7 (5'-AAGAGACGCCAAGAGGTTTATCC-3', 5'-CGCACGCTGGAGACGAAT $\left.-3^{\prime}\right)$; and TK1 (5'-TGAGAGGTGGGACGAGTCTTG-3', 5'-TGCCCGCGAACTCA AAA-3').

\section{Conflict of Interest}

The authors declare no conflict of interest.

Acknowledgements. We thank Rafael Aldabe (University of Navarra, Pamplona, Spain) for donating the Ad-TK virus and Milena Grossi for helpful discussion. This work has been supported by grant no. RF-2010-2310869 from the Italian Ministry of Health to DP, AIRC grant no. IG 2012N.12746 to MC, and by Telethon grant no. GGP14005 and AIRC grant no. IG15818 to VB.

1. Blau HM, Pomerantz JH. Re"evolutionary" regenerative medicine. JAMA 2011; 305: 87-88 2. Yaffe $D$, Gershon D. Multinucleated muscle fibres: induction of DNA synthesis and mitosis by polyoma virus infection. Nature 1967; 215: 421-424.

3. Fogel M, Defendi V. Infection of muscle cultures from various species with oncogenic DNA viruses (sv40 and polyoma). Proc Natl Acad Sci USA 1967; 58: 967-973.

4. Wang $H$, Loof S, Borg P, Nader GA, Blau HM, Simon A. Turning terminally differentiated skeletal muscle cells into regenerative progenitors. Nat Commun 2015; 6: 7916.

5. Crescenzi M, Soddu S, Tato F. Mitotic cycle reactivation in terminally differentiated cells by adenovirus infection. J Cell Physiol 1995; 162: 26-35.

6. Latella L, Sacco A, Pajalunga D, Tiainen M, Macera D, D'Angelo M et al. Reconstitution of cyclin d1-associated kinase activity drives terminally differentiated cells into the cell cycle. Mol Cell Biol 2001; 21: 5631-5643.

7. Hjiantoniou E, Anayasa M, Nicolaou P, Bantounas I, Saito M, Iseki S et al. Twist induces reversal of myotube formation. Differentiation 2008; 76: 182-192.

8. Pajalunga D, Mazzola A, Salzano AM, Biferi MG, De Luca G, Crescenzi M. Critical requirement for cell cycle inhibitors in sustaining nonproliferative states. J Cell Biol 2007; 176: $807-818$

9. Blais A, van Oevelen CJ, Margueron R, Acosta-Alvear D, Dynlacht BD. Retinoblastoma tumor suppressor protein-dependent methylation of histone h3 lysine 27 is associated with irreversible cell cycle exit. J Cell Biol 2007; 179: 1399-1412.

10. Endo T, Nadal-Ginard B. Reversal of myogenic terminal differentiation by sv40 large $t$ antigen results in mitosis and apoptosis. J Cell Sci 1998; 111: 1081-1093.

11. Pajalunga D, Puggioni EM, Mazzola A, Leva V, Montecucco A, Crescenzi M. DNA replication is intrinsically hindered in terminally differentiated myotubes. PLOS ONE 2010; 5: e11559.

12. Gandhi VV, Samuels DC. A review comparing deoxyribonucleoside triphosphate (dntp) concentrations in the mitochondrial and cytoplasmic compartments of normal and transformed cells. Nucleosides Nucleotides Nucleic Acids 2011; 30: 317-339.

13. Rampazzo C, Miazzi C, Franzolin E, Pontarin G, Ferraro P, Frangini M et al. Regulation by degradation, a cellular defense against deoxyribonucleotide pool imbalances. Mutat Res 2010; 703: 2-10.
14. Reichard P. Interactions between deoxyribonucleotide and DNA synthesis. Annu Rev Biochem 1988; 57: 349-374.

15. Hu CM, Chang ZF. Mitotic control of dttp pool: a necessity or coincidence? J Biomed Sci 2007; 14: 491-497.

16. Ferraro P, Pontarin G, Crocco L, Fabris S, Reichard P, Bianchi V. Mitochondrial deoxynucleotide pools in quiescent fibroblasts: a possible model for mitochondrial neurogastrointestinal encephalomyopathy (mngie). J Biol Chem 2005; 280: 24472-24480.

17. Frangini M, Franzolin E, Chemello F, Laveder P, Romualdi C, Bianchi V et al. Synthesis of mitochondrial DNA precursors during myogenesis, an analysis in purified c2c12 myotubes. J Biol Chem 2013; 288: 5624-5635.

18. Milasincic DJ, Dhawan J, Farmer SR. Anchorage-dependent control of muscle-specific gene expression in c2c12 mouse myoblasts. In Vitro Cell Dev Biol Anim 1996; 32: 90-99.

19. Franzolin E, Pontarin G, Rampazzo C, Miazzi C, Ferraro P, Palumbo E et al. The deoxynucleotide triphosphohydrolase samhd1 is a major regulator of DNA precursor pools in mammalian cells. Proc Natl Acad Sci USA 2013; 110: 14272-14277.

20. Lahouassa H, Daddacha W, Hofmann H, Ayinde D, Logue EC, Dragin L et al. Samhd1 restricts the replication of human immunodeficiency virus type 1 by depleting the intracellular pool of deoxynucleoside triphosphates. Nat Immunol 2012; 13: 223-228.

21. Palumbo E, Matricardi L, Tosoni E, Bensimon A, Russo A. Replication dynamics at common fragile site fra6e. Chromosoma 2010; 119: 575-587.

22. Blow JJ, Ge XQ. A model for DNA replication showing how dormant origins safeguard against replication fork failure. EMBO Rep 2009; 10: 406-412.

23. Lebofsky R, Heilig R, Sonnleitner M, Weissenbach J, Bensimon A. DNA replication origin interference increases the spacing between initiation events in human cells. Mol Biol Cell 2006; 17: 5337-5345.

24. Palumbo E, Tosoni E, Russo A. General and specific replication profiles are detected in normal human cells by genome-wide and single-locus molecular combing. Exp Cell Res 2013; 319: 3081-3093.

25. Håkansson $\mathrm{P}$, Hofer $\mathrm{A}$, Thelander $\mathrm{L}$. Regulation of mammalian ribonucleotide reduction and dntp pools after DNA damage and in resting cells. J Biol Chem 2006; 281 : 7834-7841.

26. Nordlund P, Reichard P. Ribonucleotide reductases. Annu Rev Biochem 2006; 75: 681-706.

27. Coppock DL, Pardee AB. Control of thymidine kinase mrna during the cell cycle. Mol Cell Biol 1987; 7: 2925-2932

28. Travali S, Lipson KE, Jaskulski D, Lauret E, Baserga R. Role of the promoter in the regulation of the thymidine kinase gene. Mol Cell Biol 1988; 8: 1551-1557.

29. Camarda G, Siepi F, Pajalunga D, Bernardini C, Rossi R, Montecucco A et al. A prb-independent mechanism preserves the postmitotic state in terminally differentiated skeletal muscle cells. J Cell Biol 2004; 167: 417-423.

30. Agalioti T, Chen G, Thanos D. Deciphering the transcriptional histone acetylation code for a human gene. Cell 2002; 111: 381-392.

31. Egloff S, Dienstbier M, Murphy S. Updating the rna polymerase ctd code: adding genespecific layers. Trends Genet 2012; 28: 333-341.

32. Roizman B, Knipe DM. Herpesviridae. In: Knipe DM, Howley PM (eds). Virology, 4th edn. Lippincott Williams \& Wilkins: Philadelphia, PA, USA, 2001, pp 2399-2459.

33. Jasmer DP. Trichinella spiralis infected skeletal muscle cells arrest in $\mathrm{g} 2 / \mathrm{m}$ and cease muscle gene expression. J Cell Biol 1993; 121: 785-793.

34. Rando TA, Blau HM. Primary mouse myoblast purification, characterization, and transplantation for cell-mediated gene therapy. J Cell Biol 1994; 125: 1275-1287.

35. Bianchi V, Fortunati E. Cellular effects of an anionic surfactant detected in v79 fibroblasts by different cytotoxicity tests. Toxicol In Vitro 1990; 4: 9-16.

36. Bader D, Masaki T, Fischman DA. Immunochemical analysis of myosin heavy chain during avian myogenesis in vivo and in vitro. J Cell Biol 1982; 95: 763-770.

37. Diffley JF. Quality control in the initiation of eukaryotic DNA replication. Philos Trans $R$ Soc Lond B Biol Sci 2011; 366: 3545-3553.

38. Franzolin E, Rampazzo C, Perez-Perez MJ, Hernandez Al, Balzarini J, Bianchi V. Bromovinyl-deoxyuridine: a selective substrate for mitochondrial thymidine kinase in cell extracts. Biochem Biophys Res Commun 2006; 344: 30-36.

39. Gurtner A, Manni I, Fuschi P, Mantovani R, Guadagni F, Sacchi A et al. Requirement for down-regulation of the ccaat-binding activity of the nf-y transcription factor during skeletal muscle differentiation. Mol Biol Cell 2003; 14: 2706-2715.

40. Gurtner A, Fuschi P, Magi F, Colussi C, Gaetano C, Dobbelstein M et al. Nf-y dependent epigenetic modifications discriminate between proliferating and postmitotic tissue. PLOS ONE 2008; 3: e2047.

41. Bianchi V, Pontis E, Reichard P. Regulation of pyrimidine deoxyribonucleotide metabolism by substrate cycles in dcmp deaminase-deficient v79 hamster cells. Mol Cell Biol 1987; 7: $4218-4224$.

Supplementary Information accompanies this paper on Cell Death and Differentiation website (http://www.nature.com/cdd) 